\title{
EFECTOS DEL ENTRENAMIENTO AERÓBICO VERSUS FORTALECIMIENTO DE SOBRECARGA MUSCULAR EN PACIENTES CON DIABETES MELLITUS TIPO 2 EN UN CONTEXTO DE ATENCIÓN PRIMARIA CHILENA
}

\section{EFFECTS OF AEROBIC TRAINING VERSUS MUSCULAR STRENGTHENING OVERLOAD IN PATIENTS WITH DIABETES MELLITUS TYPE 2 IN CONTEXT OF CHILEAN PRIMARY CARE}

\author{
Peñailillo, Luis*; Armijo, Felipe**; Osorio, Mireya** \& Rosales, Jaime** \\ *Klgo., MSc, Escuela de Kinesiología, Pontificia Universidad Católica de Valparaíso, Valparaíso, Chile. \\ **Kinesiólogo
}

PEÑAlliLlO, L.; ARMiJO, F.; OSORIO, M.; ROSALES, J. Efectos del entrenamiento aeróbico versus fortalecimiento de sobrecarga muscular en pacientes con Diabetes Mellitus Tipo 2 en un contexto de atención primaria chilena. Rev. Motr. Hum., 11(1): 11-22, 2010.

\begin{abstract}
RESUMEN
Comparar dos protocolos de entrenamiento, aeróbico y sobrecarga, de ocho semanas de duración en relación a los cambios producidos en variables fisiológicas y kineantropométricas de pacientes adultos con Diabetes Mellitus tipo 2 (DM2) pertenecientes al Centro de Salud Laurita Vicuña, comuna El Bosque, Santiago.

La muestra quedó conformada por 15 sujetos distribuidos aleatoriamente en 3 grupos: Aeróbico ( $n=5)$, que realizó entrenamiento en bicicleta estática a una intensidad moderada (50-70\% de la frecuencia cardiaca máxima (FCmáx)); Sobrecarga (n=5), que realizó entrenamiento al 70-80\% de 1 RM; y Control ( $n=5)$, que no realizó entrenamiento. Se evaluó la glicemia capilar, una vez por semana, antes y después de cada sesión. Se evaluaron las variables Hemoglobina Glicosilada (HbAlc), glicemia en ayunas y kineantropométricas al inicio y término de la intervención.

Ambos grupos de entrenamiento obtuvieron disminuciones no significativas de la HbAlc. El grupo Aeróbico disminuyó significativamente $(p=0,04)$ su glicemia capilar en la sesión de ejercicio, glicemia en ayunas, circunferencia de cintura y presentó una disminución porcentual y absoluta del tejido adiposo; el grupo de Sobrecarga disminuyó significativamente $(p=0,04)$ el tejido adiposo en valor absoluto y los pliegues cutáneos centrales. Todos los grupos aumentaron su masa muscular, siendo significativo ( $p=0,04)$ para los grupos Aeróbico y Control.

Sólo el grupo Aeróbico obtuvo cambios significativos tanto en variables relacionadas al control glicémico, como a la composición y distribución corporal, pero al comparar ambos grupos de entrenamiento no podemos concluir que un protocolo produzca mayores cambios respecto al otro.
\end{abstract}

Palabras Clave: Ejercicio - resistencia a la insulina - hemoglobina glicosilada - control glicémico, circunferencia de cintura - kineantropometría.

\section{INTRODUCCIÓN}

La Diabetes Mellitus tipo 2 (DM2) es una de las patologías que genera mayor discapacidad y mortalidad en el mundo, ocupando gran parte de los recursos sanitarios en todos los países. En Chile, constituye un importante problema de salud, situación que no difiere de otros países latinoamericanos debido a que su población se ve enfrentada a los mismos factores de riesgo que han aumentado su prevalencia a nivel mundial $(1,2)$.

Está demostrado que el ejercicio es una medida efectiva para mejorar parámetros de control glicémico, como la hemoglobina glicosilada (HbA1c), la mortalidad y el riesgo total de enfermedades cardiovasculares $(3,4,5,6,7,8)$. El entrenamiento sistemático puede llegar a extender el efecto agudo sobre la glicemia mediado por el ejercicio, lo que finalmente se traducirá en cambios favorables para el control glicémico. Además, el entrenamiento es capaz de producir cambios metabólicos a largo plazo que serán reflejados en la composición corporal, tales como la preservación de la masa muscular y la reducción de la masa grasa total y abdominal. Así, estos cambios que se podrían relacionar al beneficio glucorregulatorio del entrenamiento y a el riesgo de enfermedades cardiovasculares $(9,10)$.

A pesar de la evidencia internacional que respalda al entrenamiento físico como parte fundamental del tratamiento de pacientes con DM2, no existe una unificación de criterios en relación a éste, ni claridad acerca del tipo de entrenamiento más recomendable para alcanzar metas de control glicémico. Por esta razón, este estudio tiene como propósito comparar el efecto de dos protocolos de entrenamiento: uno aeróbico de resistencia versus otro basado en el fortalecimiento muscular, evaluando variables fisiológicas y de composición corporal. 
PEÑAILILlO, L.; ARMIJO, F.; OSORIO, M.; ROSALES, J. Efectos del entrenamiento aeróbico versus fortalecimiento de sobrecarga muscular en pacientes con Diabetes Mellitus Tipo 2 en un contexto de atención primaria chilena. Rev. Motr. Hum., 11(1): 11-19, 2010.

\section{SUJETOS Y MÉTODOS}

Se seleccionaron 15 pacientes de sexo femenino, con diagnóstico de DM2 pertenecientes a un centro de atención primaria del sector oriente de Santiago, Chile. Como criterio de inclusión se consideró a pacientes adultos menores de 65 años de edad que contaran con el diagnóstico de DM2, según los criterios establecidos en la Guía Clínica de DM2 del MINSAL, HbA1c sobre el 6,1\%, no insulinorrequirentes y que contaran con un electrocardiograma normal (11). Los datos generales de la muestra se muestran en la Tabla 1, previa firma del consentimiento informado aprobado por la comisión de bioética de la Pontificia Universidad Católica de Valparaíso. Los sujetos fueron divididos aleatoriamente en tres grupos: grupo de entrenamiento Aeróbico (A), grupo de entrenamiento de Sobrecarga (S) y grupo Control (C).

\begin{tabular}{cccc} 
& Control & Aeróbico & Sobrecarga \\
\cline { 2 - 4 } $\mathbf{n}$ & 5 & 5 & 5 \\
Edad (años) & $52,8( \pm 9,09)$ & $56,4( \pm 7,1)$ & $49,2( \pm 12,8)$ \\
Años de DM2 (años) & $6,7( \pm 8,7)$ & $9,5( \pm 9,8)$ & $3,4( \pm 2,8)$ \\
Peso (Kg) & $80,9( \pm 13,3)$ & $71,3( \pm 11,5)$ & $67,8( \pm 16,2)$ \\
Talla (cm) & $159,3( \pm 6,7)$ & $152,3( \pm 6,7)$ & $151,2( \pm 5,8)$ \\
IMC (kg/ $\left.\mathbf{m}^{2}\right)$ & $31,7( \pm 4,6)$ & $30,7( \pm 4,3)$ & $29,5( \pm 5,6)$
\end{tabular}

Tabla 1: Datos Generales de la Muestra.

Los participantes se sometieron a evaluaciones basales de las variables kineantropométricas, muestras de sangre y de 10 repeticiones máximas una semana previa al comienzo del entrenamiento. Luego, los participantes fueron sometidos a ocho semanas de entrenamiento, para finalizar con las evaluaciones post-entrenamiento a lo menos 72 horas después de la última sesión de entrenamiento, para eliminar efectos agudos del ejercicio.

Los sujetos fueron planificados para entrenar tres sesiones por semana, con una duración aproximada de 60 minutos cada una, por un período de ocho semanas de entrenamiento más una primera semana de adaptación y familiarización. Cada sesión se inició con un calentamiento grupal, agregando ejercicios respiratorios y de elongación para todos los grupos musculares involucrados. Las sesiones de ambos grupos fueron supervisados por al menos dos investigadores. Los participantes del protocolo Aeróbico realizaron un entrenamiento en bicicleta estática. La intensidad de trabajo se determinó según la fórmula de Karvonen (12), estableciendo una intensidad moderada, entre el 50\% y $70 \%$ de la FCmáx5. La frecuencia cardiaca (FC) se obtuvo mediante un monitor de frecuencia cardiaca (Polar, RS-200, Finlandia). Al finalizar la cuarta semana del período de entrenamiento, se evaluó nuevamente la FC objetivo para modificar sus valores individuales de carga.

Para este grupo, el período total de ocho semanas se dividió en cuatro períodos de dos semanas cada uno, diferenciados en cantidad y distribución del volumen de trabajo semanal, planificados según principios del entrenamiento. Los participantes de este grupo realizaron nueve ejercicios que se enfocaban a diferentes grupos musculares, a través de la utilización de peso corporal y pesos libres. Para los ejercicios que utilizan cargas externas se utilizó una evaluación submaximal de la repetición máxima (RM) mediante la fórmula de Brzicki (13), con el fin de disminuir el riesgo de lesiones; para los ejercicios que utilizan el peso corporal se utilizó el número máximo de repeticiones hasta el momento que el individuo no podía realizar otra repetición por fatiga o cuando distorsionaba el gesto técnico durante su ejecución. El entrenamiento se planificó utilizando el $70-75 \%$ del RM y de 12 a 15 repeticiones según la pirámide de equivalencias de Tudor Bompa (14). A la cuarta semana se reevaluaron las cargas de entrenamiento para ser fijadas al $80 \%$ RM.

Las variables fueron evaluadas mediante exámenes de laboratorio, con muestras sanguíneas obtenidas una semana antes y una semana posterior al período de entrenamiento, en el mismo centro de salud al cual pertenece la muestra. La respuesta aguda de la glicemia al ejercicio se refiere a los cambios que produce una sesión de ejercicios en la glicemia plasmática. Ambas variables se midieron a través de las glicemias capilares, evaluadas una vez a la semana antes y después de la sesión, en $\mathrm{mg} / \mathrm{dl}$, con un medidor de glucosa portátil (Accucheck Active, USA).

Para las variables kineantropométricas se realizaron medidas correspondientes a un perfil kineantropométrico restringido, según el protocolo definido por la International Society of Advances in Kineanthropometry (I.S.A.K.). La evaluación fue llevada a cabo por kineantropometristas certificados nivel 1 I.S.A.K., utilizándose kits antropométricos calibrados 
PEÑAILILlO, L.; ARMIJO, F.; OSORIO, M.; ROSALES, J. Efectos del entrenamiento aeróbico versus fortalecimiento de sobrecarga muscular en pacientes con Diabetes Mellitus Tipo 2 en un contexto de atención primaria chilena. Rev. Motr. Hum., 11(1): 11-19, 2010.

(Rosscraft®, Canadá). Se consideró un error de medición inferior al 5\% en los pliegues cutáneos, y de $1 \%$ para el resto de las mediciones. La evaluación fue realizada al inicio y al final del entrenamiento por el mismo evaluador, utilizando el mismo kit kineantropométrico y a la misma hora de la evaluación anterior, con el fin de mantener la validez. Para obtener el fraccionamiento corporal en cinco componentes se utilizaron las ecuaciones propuestas por Deborah Kerr (15). La masa grasa y masa muscular fueron operacionalizadas en valor absoluto $(\mathrm{kg})$ y en porcentaje, para una mejor interpretación de los resultados. Masa ósea, vísceras y piel, no mostraron diferencias y los resultados no serán mostrados.

Se utilizan las siguientes ecuaciones:

$$
\begin{gathered}
\text { S ADIP }=\text { sumatoria } \\
(\text { TPSF+SSSF+SISF+ABSF+THSF }+ \text { MCSF }) \\
\text { Z ADIP }=[\text { S ADIP } *(170,18 / H T)-116,41] / 34,79
\end{gathered}
$$

Donde: $\mathrm{HT}=$ altura en $\mathrm{cm} ; 116,41=$ sumatoria de medidas Phantom de los pliegues cutáneos; 34,79= sumatoria de los desvíos estándar Phantom para los pliegues cutáneos; TPSF= pliegue cutáneo de tríceps; $\mathrm{SSSF}=$ pliegue cutáneo subescapular; $\mathrm{SISF}=$ pliegue cutáneo supra espinal; $\mathrm{ABSF}=$ pliegue cutáneo abdominal; THSF= pliegue cutáneo frontal del muslo; $\mathrm{MCSF}=$ pliegue cutáneo de la pantorrilla media .

$$
\operatorname{MADIP}(\mathrm{Kg} .)=\left[\left(\mathrm{Z} \text { ADIP*5, 85)+25,6]/ (170,18/HT) }{ }^{3}\right.\right.
$$

Donde: $\mathrm{M}$ ADIP = masa adiposa $(\mathrm{en} \mathrm{kg}) ; \mathrm{Z}$ ADIP $=$ score de proporcionalidad Phantom para la masa adiposa; $25,6=$ constante del método para medida de masa adiposa Phantom (en $\mathrm{kg}) ; 5,85=$ constante del método para desvío estándar de la masa adiposa Phantom (en $\mathrm{kg}$ ).

\section{S MUS $=$ sumatoria $($ P ARC + P FA + PTHC + PMCC + P CHC)}

$$
\text { Z MUS }=[S \text { MUS* }(170,18 / H T)-207,21] / 13,74
$$

Donde: $207,21=$ sumatoria de las medidas Phantom de los perímetros corregidos; $13,74=$ sumatoria de los desvíos estándar Phantom para los perímetros corregidos; PARC $=$ perímetro del brazo (relajado), corregido por el pliegue cutáneo del tríceps; P FA=perímetro del antebrazo; P THC= perímetro del muslo, corregido por el pliegue cutáneo del muslo frontal; $\mathrm{P} \mathrm{MCC}=$ perímetro de una pantorrilla, corregido por el pliegue cutáneo de la pantorrilla medial; $\mathrm{P}$ $\mathrm{CHC}=$ perímetro de la caja torácica, corregido por el pliegue cutáneo subescapular.

$$
\operatorname{M~MUS~}(\mathrm{kg})=\left[\left(\mathrm{Z} \text { MUS*5,4)+24,5] } /(170,18 / \mathrm{HT})^{3}\right.\right.
$$

Donde: $\mathrm{M}$ MUS= masa muscular $(\mathrm{en} \mathrm{kg}) ; \mathrm{Z}$ MUS= score de proporcionalidad Phantom para masa muscular; 24,5= constante del método para media de masa muscular Phantom (en $\mathrm{kg}) ; 5,4=$ constante del método para desvío estándar Phantom para el músculo (en $\mathrm{kg}$ ).

Además, se evaluó la circunferencia de cintura (CC) que corresponde a la medición del perímetro a nivel del punto medio entre el reborde costal y la cresta ilíaca de ambos lados en centímetros y se encuentra dentro de las medidas realizadas en el perfil restringido (16).

Como variables intervinientes se consideraron los fármacos hipoglicemiantes orales utilizados en el tratamiento de la DM2 y la Ingesta Calórica Diaria, que es el promedio de las calorías ingeridas al día por cada participante. Esta variable fue controlada mediante una bitácora alimenticia que registraba lo ingerido el día anterior de cada sesión, no mostrando diferencias significativas entre el consumo en kilocalorías diarias.

Para todas las variables estudiadas se realizó estadística descriptiva. En el análisis intragrupo se utilizó estadística no paramétrica, a través del test de rangos con signos de Wilcoxon. En el análisis de variables intergrupo se utilizó la prueba de Kruskal- Wallis, donde los resultados fueron considerados significativos para valores de $\mathrm{p}<0.05$. Se utilizó el software de análisis estadístico Stata ${ }^{\mathrm{TM}}$ 10.0.

\section{RESULTADOS}

La adhesión de la muestra inicial, expresada como el porcentaje de sujetos que finalizaron la intervención, fue mayor para los grupos A y S con un $83,33 \%$ para cada uno y un $71,43 \%$ para el grupo C. La asistencia a las sesiones de entrenamiento para ambos grupos de ejercicio fue de $85,81 \pm 8 \%$. La asistencia fue de un $89,14 \pm 8,6 \%$ en el grupo A y un $82,48 \pm 7,4 \%$ en el grupo $\mathrm{S}$.

En la Figura 1 se observa el comportamiento semanal de la variable glicemia capilar en reposo, evaluada una vez por semana en los grupos de ejercicio. La comparación intragrupo de la primera con la octava semana no presentó diferencias estadísticamente significativas al finalizar la intervención, para ninguno de los grupos. En el grupo A se observa una disminución no significativa de la glicemia capilar en reposo $(p=0,07)$ entre la primera y octava semana. El análisis intergrupo entre la primera, cuarta y octava semana, no presentó diferencias estadísticamente significativas. Del análisis de la respuesta aguda de la glicemia al ejercicio podemos observar, en primer lugar, que ambos grupos tienen un valor de glicemia inicial similar de 141,04 para el grupo Aeróbico y 142,77 mg/dl para el grupo de Sobrecarga. En la comparación intragrupo de esta variable sólo el grupo Aeróbico presenta una disminución significativa de 33,6 $\mathrm{mg} / \mathrm{dl}(\mathrm{p}=0,43)$ en su glicemia capilar después del ejercicio y el grupo de Sobrecarga obtiene una disminución de 14,9 $\mathrm{mg} / \mathrm{dl}(\mathrm{p}=0,22)$ (Figura 1). 
PEÑAILILlO, L.; ARMIJO, F.; OSORIO, M.; ROSALES, J. Efectos del entrenamiento aeróbico versus fortalecimiento de sobrecarga muscular en pacientes con Diabetes Mellitus Tipo 2 en un contexto de atención primaria chilena. Rev. Motr. Hum., 11(1): 11-19, 2010.

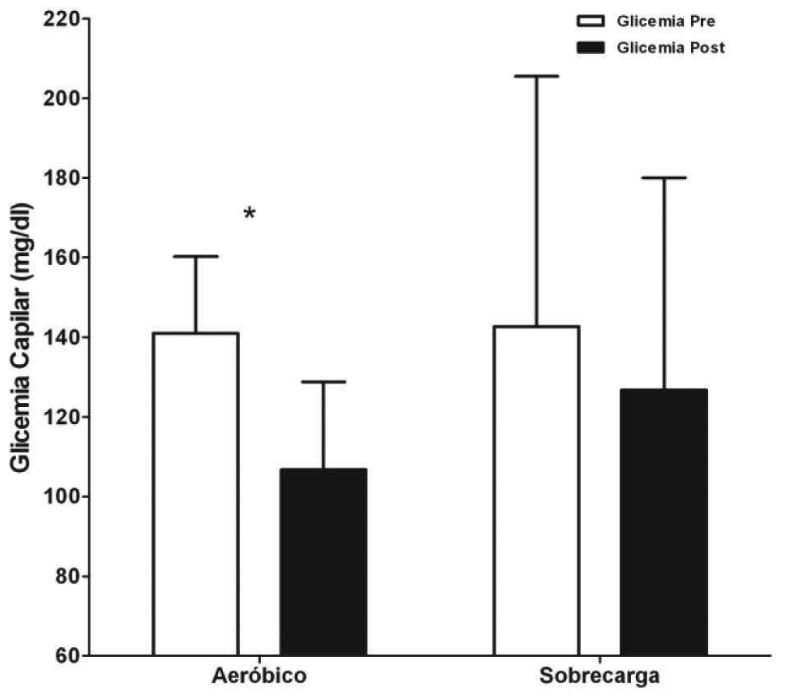

Figura 1: Glicemia Post ejercicio.

Al evaluar los tres grupos de estudio antes y después de la intervención, los resultados de la HbA1c muestran que en el grupo Aeróbico hubo una disminución promedio de $0,6 \%$ $(\mathrm{p}=0,14)$; en el grupo Sobrecarga una disminución promedio de $0,3 \%(p=0,17)$; mientras en el grupo $\mathrm{C}$ aumentó en promedio $0,08 \%(p=0,78)$ (Tabla 3$)$. Al comparar el comportamiento intergrupo de la HbAlc no se encontraron diferencias estadísticamente significativas al finalizar la intervención (Figura 2).

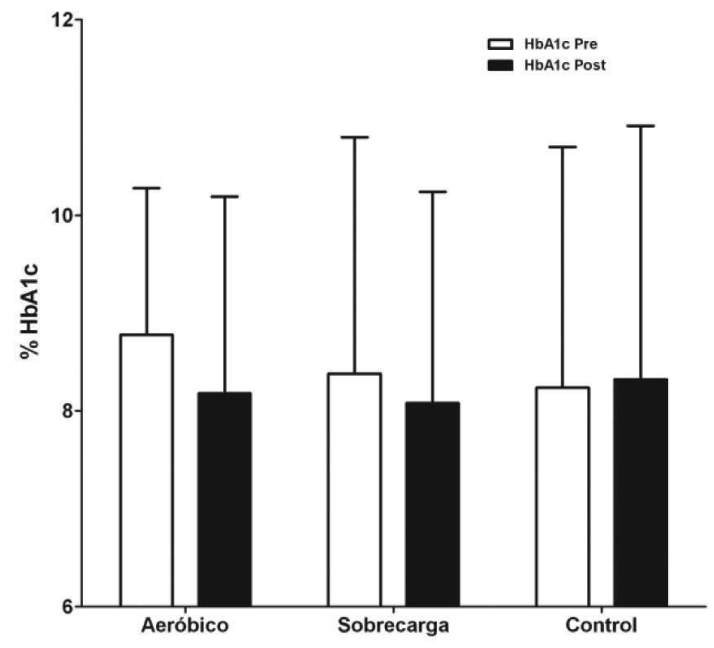

Figura 2: Hemoglobina Glicosilada.
En relación a la glicemia en ayunas, el grupo Aeróbico mostró una disminución promedio de $36 \mathrm{mg} / \mathrm{dl}(\mathrm{p}=0,04)$, siendo el único grupo que mostró significancia estadística en la comparación intragrupo. Al contrastar el comportamiento intergrupo de esta variable no se encontraron diferencias estadísticamente significativas al finalizar la intervención (Figura 3).

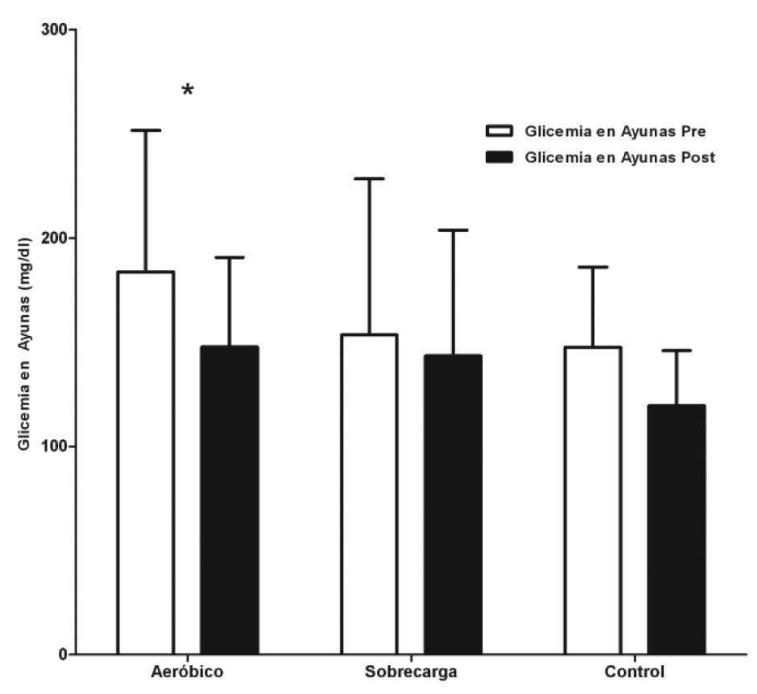

Figura 3: Glicemia en Ayunas.

Con respecto al IMC, sólo el grupo A presentó una disminución estadísticamente significativa $(p=0.04)$ mostrando una disminución promedio de $0.44 \mathrm{~kg} / \mathrm{m}^{2}$, comparando los valores previos con los de post intervención (Figura 4).

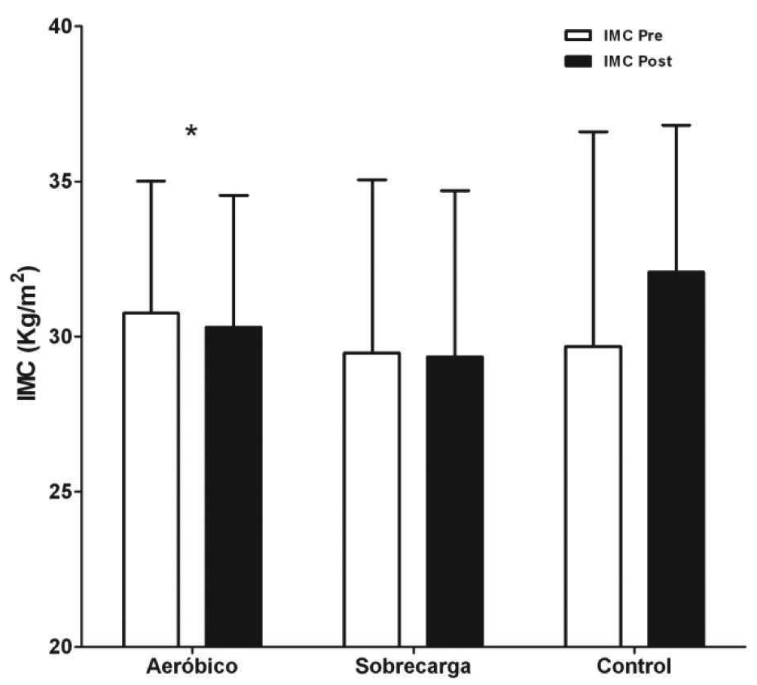

Figura 4: Índice de Masa Corporal. 
PEÑAILILlO, L.; ARMIJO, F.; OSORIO, M.; ROSALES, J. Efectos del entrenamiento aeróbico versus fortalecimiento de sobrecarga muscular en pacientes con Diabetes Mellitus Tipo 2 en un contexto de atención primaria chilena. Rev. Motr. Hum., 11(1): 11-19, 2010.

En relación al porcentaje de masa grasa, el grupo Aeróbico mostró una disminución de $2,75 \%$ con respecto al total de grasa ( $\mathrm{p}=0,04)$, siendo el único grupo que mostró significancia estadística en la comparación intragrupo (Figura 5).

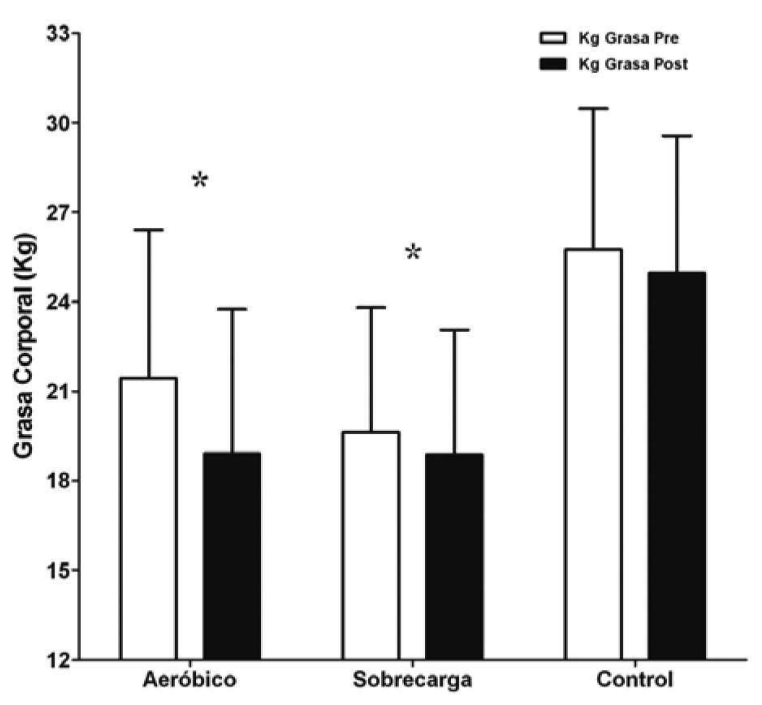

Figura 5. Porcentaje de Masa Grasa.

En relación a la masa grasa en valor absoluto, el grupo A mostró una disminución de $2,54 \mathrm{~kg}(\mathrm{p}=0,04)$ y el grupo $\mathrm{S}$ una disminución de $0,76 \mathrm{~kg}(\mathrm{p}=0,04)$, siendo para ambos grupos una reducción estadísticamente significativa en la comparación intragrupo. Además, en el porcentaje de masa muscular el grupo A mostró un aumento de $1,61 \%(\mathrm{p}=0,04)$, el grupo $\mathrm{S}$ aumentó un $1,4 \%(\mathrm{p}=0,07)$, mientras el grupo $\mathrm{C}$ mostró un aumento de $1,07 \%(\mathrm{p}=0,04)$. En la comparación intragrupo, los grupos A y C mostraron cambios estadísticamente significativos (Figura 6). En la masa muscular en valor absoluto, el grupo A mostró un aumento de $0,27 \mathrm{~kg}(\mathrm{p}=0,22)$, el grupo $\mathrm{S}$ aumentó un $0,57 \mathrm{~kg}(\mathrm{p}=0,34)$, mientras el grupo $\mathrm{C}$ mostró un aumento de $1,18 \mathrm{~kg}(\mathrm{p}=0,13)$, no existiendo significancia estadística intragrupo para ninguno de ellos.

Respecto a la CC, en el grupo A disminuyó en 1,93 cm $(\mathrm{p}=0,04)$ siendo el único grupo que mostró significancia estadística en la comparación intragrupo (Figura 6).

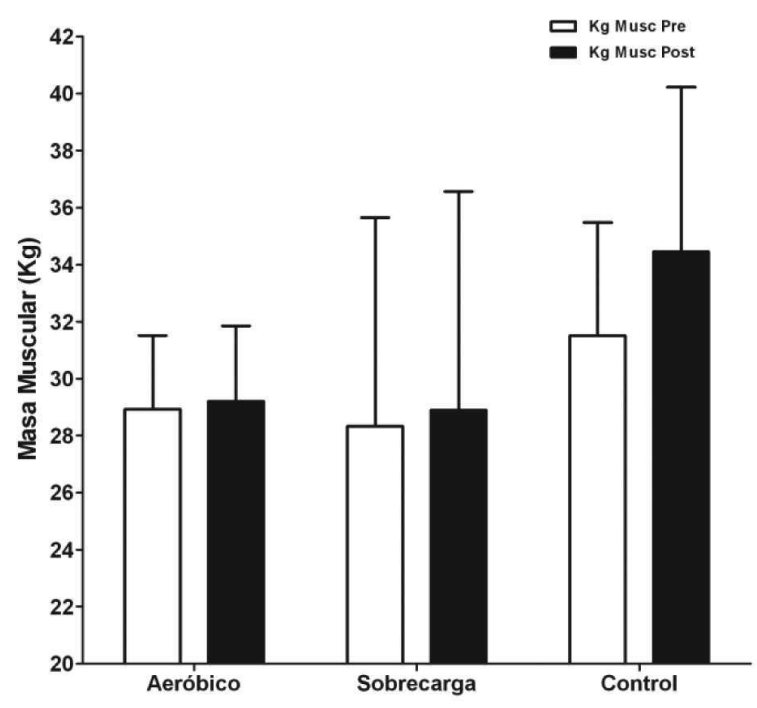

Figura 6: Porcentaje de Masa Muscular.

\section{DISCUSIÓN}

El mayor hallazgo del presente estudio fue que, mediante la aplicación de programas de entrenamiento físico en un consultorio de atención primaria, es posible reducir algunos de los marcadores de diabetes en sangre y kineantropométricos, como la glicemia en ayunas y los porcentajes de masa muscular y grasa.

El efecto agudo está representado por la variable respuesta aguda de la glicemia al ejercicio. Se ha demostrado que este efecto glucorregulatorio está relacionado a la activación prolongada del transporte de glucosa, inducido por la contracción muscular $(5,6,9)$. Los estudios con programas de ejercicio supervisado, generalmente, no caracterizan la evolución de la respuesta de la glucosa al ejercicio en el curso de un programa de entrenamiento; sin embargo, este efecto es importante si consideramos que, según la evidencia revisada, puede ser mantenido entre 24 a 48 horas después de terminado el ejercicio $(5,6,9)$.

El grupo Aeróbico tuvo una disminución significativa de $33.6 \mathrm{mg} / \mathrm{dl}$ en su glicemia plasmática. Esto concuerda con evidencia reciente de Nguyen y cols (17), que determinó la respuesta aguda de la glicemia a un plan de entrenamiento aeróbico de intensidad moderada. Ambos coinciden en la intensidad moderada utilizada, la cual logra activar el sistema de transporte de glucosa en la musculatura esquelética y, al mismo tiempo, evita la liberación masiva de hormonas contrarregulatorias, las cuales se liberan ante ejercicios de alta intensidad $(6,7)$. Estas hormonas provocan un efecto hiperglicemiante, que pudo evitarse en el protocolo Aeróbico; no así en el de Sobrecarga, que tuvo una disminución no significativa de la glicemia, al compararse los efectos pre y post entrenamiento.

El efecto crónico del ejercicio sobre el control glicémico 
PEÑAILILlO, L.; ARMIJO, F.; OSORIO, M.; ROSALES, J. Efectos del entrenamiento aeróbico versus fortalecimiento de sobrecarga muscular en pacientes con Diabetes Mellitus Tipo 2 en un contexto de atención primaria chilena. Rev. Motr. Hum., 11(1): 11-19, 2010.

está representado por la HbAlc y la glicemia en ayunas. La $\mathrm{HbA} 1 \mathrm{c}$ es el mejor indicador del control glicémico a largo plazo, por lo cual destacamos la mayor tendencia a disminuir en el grupo Aeróbico, en $0,6 \%$, que se acerca bastante a las disminuciones promedio observadas en el meta análisis de Boulé y cols (4), con una disminución promedio de $0.66 \%$; y a lo observado en dos meta análisis publicados en el año $2006(3,18)$, con disminuciones significativas de $0,5 \%$ y $0,7 \%$, respectivamente. Estos meta análisis consideran como clínicamente significativas estas disminuciones, ya que la evidencia señala que incluso una reducción modesta de la HbAlc tiene el potencial para prevenir muertes por complicaciones asociadas a la diabetes, como las enfermedades cardiovascular y cerebrovascular, que representan el 50-60\% de toda la mortalidad en poblaciones diabéticas (18). Por cada $0,5 \%$ de reducción de la $\mathrm{HbA} 1 \mathrm{c}$, se reduce el riesgo de complicaciones por DM2, tanto micro como macro vasculares en un $10,5 \%$ y reduce el riesgo de muerte asociado a DM2 en un 21\% (19).

La mayor disminución en el grupo Aeróbico puede estar ligada al resultado de su respuesta aguda de la glicemia al ejercicio, que dura lo suficiente para acoplarse al efecto agudo de una sesión subsiguiente. Esto recalca la importancia de realizar ejercicio físico regular en el paciente diabético. Sin embargo, la disminución observada en el grupo de Sobrecarga de $0.3 \%$, se aleja un poco de lo observado en el meta análisis de Snowling y cols (3), con una disminución promedio de $0.5 \%$. Dunstan y cols (20) aplicaron un protocolo de sobrecarga en pacientes con DM2 utilizando una intensidad similar a la de este estudio, mostrando una disminución promedio de HbA1c de $0,5 \%$ a los tres meses, llegando a $0,8 \%$ a los seis meses de duración. En relación a estos resultados, podemos teorizar que el menor impacto observado en la reducción de la HbA1c del grupo de Sobrecarga podría estar asociado a la menor duración de la intervención. Respecto a la glicemia en ayunas, la disminución observada en el grupo Aeróbico supera ampliamente a la disminución promedio observada en el meta análisis de Snowling y cols (3) para el mismo tipo de intervenciones. No obstante, este examen, pese a ser ampliamente utilizado en clínica y en los estudios como parámetro de control glicémico (4), no considera la variabilidad diaria de la glicemia durante el día, ya que refleja la glicemia de sólo un momento en específico, por lo que puede no tener la misma validez para determinar el control glicémico durante las ocho semanas que dura el entrenamiento. Esto no implica que su disminución no reporte beneficios, ya que, según Coutinho y cols (21), existe una relación exponencial entre las concentraciones de glicemia en ayunas y la incidencia de eventos cardiovasculares, por lo que una disminución de esta variable puede reducir el riesgo de enfermedad cardiovascular considerablemente. Con respecto a las variables kineantropométricas, el ACSM (6) considera que una reducción del peso corporal en un 10$15 \%$ podría ayudar a lograr cambios metabólicos que se traduzcan en beneficio para el control glicémico. En el presente estudio, el grupo Aeróbico fue el único que tuvo reducciones significativas en el peso corporal, reducción que dista bastante de lo que recomienda el ACSM; y también fue el único que tuvo una reducción significativa en el IMC, pero de sólo $0.44 \mathrm{~kg} / \mathrm{m}^{2}$. Sin embargo, según lo observado en este estudio y en la evidencia revisada (22), hay cambios metabólicos importantes que ocurren antes que una pérdida de peso e IMC sea evidente. Además, el IMC es menos sensible a fluctuaciones reales del tejido adiposo (23). La masa muscular se comportó de forma similar en los grupos Aeróbico y Control, ambos tuvieron un aumento significativo de esta variable. Este resultado debe interpretarse con cautela, ya que puede ser el reflejo de un aumento de otros componentes corporales, dando la falsa impresión de un aumento de la masa muscular. Respecto del aumento no significativo en el grupo de Sobrecarga, dos estudios que utilizaron un protocolo similar al de este grupo obtuvieron aumentos significativos de la masa muscular, pero con duraciones de 12 y 16 semanas cada uno, mayor a la de nuestro protocolo de sobrecarga $(20,24)$. La evidencia señala que se necesitan al menos de tres a seis meses de entrenamiento para observar hipertrofia muscular significativa $(5,14)$. Aún así, la adaptación neuronal y metabólica ocurre antes que la hipertrofia muscular sea evidente.

La masa grasa tuvo una disminución significativa en el grupo Aeróbico; durante una sesión de ejercicio de intensidad moderada, la utilización de las fuentes energéticas es progresiva desde aquellas fuentes más inmediatas, como el glicógeno muscular y la glucosa sanguínea, hasta aquellas fuentes acumuladas en mayor cantidad, como los depósitos de tejido adiposo (25), en la medida que la duración del entrenamiento sea lo suficientemente larga (26). La evidencia revisada indica que los sujetos con insulinorresistencia, como los diabéticos, tienen menores depósitos de glicógeno muscular (27), por que se podría hipotetizar que al mantener hasta 50 minutos de entrenamiento, la intensidad moderada pudo privilegiar la utilización de fuentes energéticas más abundantes como el tejido adiposo, al privilegiar el metabolismo oxidativo característico del entrenamiento aeróbico $(28,29)$.

Un estudio de Miyazaki y cols (30) determinó una alta correlación entre concentraciones de citokinas inflamatorias con la presencia de insulinorresistencia. Otro estudio de Lastra y cols (31) determinó que, ante concentraciones de citokinas como el factor de necrosis tumoral $\alpha$ y de interleukina 6 , aumenta la producción hepática de reactantes de fase aguda, como la proteína $\mathrm{C}$ reactiva, la cual tiene una alta correlación con la presencia de enfermedades cardiovasculares. Frente a esto, la disminución de 2,54 kg de masa grasa observada en el grupo Aeróbico podría estar relacionada con la disminución de todos estos componentes, por lo tanto, de la insulinorresistencia y del riesgo de eventos cardiovasculares.

Respecto a la CC, Wahrenberg y cols (32), determinó que la $\mathrm{CC}$ es la variable que tiene mejor correlación con insulinorresistencia. 
PEÑAILILlO, L.; ARMIJO, F.; OSORIO, M.; ROSALES, J. Efectos del entrenamiento aeróbico versus fortalecimiento de sobrecarga muscular en pacientes con Diabetes Mellitus Tipo 2 en un contexto de atención primaria chilena. Rev. Motr. Hum., 11(1): 11-19, 2010.

Esta medición permite evaluar cambios en la distribución del tejido adiposo, diferenciándola de las disminuciones globales de grasa (33). Tiene una alta correlación con la grasa de distribución abdominal, la cual cobra relevancia al relacionarse con la presencia de enfermedades cardiovasculares (34) e insulinorresistencia (32), independiente del género o la raza $(35,37)$; ademá, es el índice clínico que tiene una mayor correlación con la grasa visceral estimada por métodos de imagen $(33,35,38,39)$. Respecto de la disminución significativa de $1.93 \mathrm{~cm}$ de la CC del grupo Aeróbico, un estudio reciente de Sigal y cols (40) que utilizó un protocolo aeróbico similar al de este estudio, encontró una disminución de $3 \mathrm{~cm}$; sin embargo, éste tuvo una mayor duración total respecto al nuestro. La disminución significativa de la CC en este grupo pudo estar relacionada, más que a grasa subcutánea central, a grasa intrabdominal, aspecto importante ya que contiene a la grasa del tipo visceral, que aporta un mayor contenido de ácidos grasos libres al hígado y tiene un mayor grado de secreción de citokinas inflamatorias $(22,41)$. Sin embargo, estas estimaciones sólo pueden considerarse hipotéticas, ya que se requiere de métodos de imagen o de estimación de la composición corporal más precisos, como la tomografía axial computarizada o la resonancia nuclear magnética, para describir con precisión los cambios de distribución del tejido graso (39).

\section{CONCLUSIÓN}

En vista de los resultados, el entrenamiento Aeróbico tuvo una mejoría significativa en las variables relacionadas con el control glicémico, y las relacionadas a la composición y distribución corporal, con respecto a sí mismo. Por su parte, el entrenamiento de Sobrecarga tuvo mejoría significativa sólo en una variable de composición corporal y en una de distribución. Con esto recalcamos que, pese a que hubo una periodización del entrenamiento para que ambos protocolos tuvieran un volumen similar, éstos ocupan diferentes conceptos base en su fisiología, por lo que pueden tener distinto impacto sobre las variables analizadas, lo cual pudo influir para que tuviesen distintos resultados con respecto a sí mismo.

El fraccionamiento corporal en cinco componentes es un método que tiene validación in vitro e in vivo, siendo el método sencillo y económico de obtener una estimación indirecta de la composición corporal, factible de ser aplicada en clínica.

Así, el presente estudio aporta evidencia científica acerca del efecto beneficioso del entrenamiento aeróbico y de sobrecarga en pacientes con DM2, destacando que fue realizado en un contexto de atención primaria, en una población con alto riesgo socioeconómico y que cumple con criterios internacionales de prescripción y planificación del entrenamiento.

\section{REFERENCIAS BIBLIOGRÁFICAS}

1. Wild S, Roglic G, Green A, Sicree R, King H. Global Prevalence of Diabetes, Estimates for the year 2000 and projections for 2030. Diabetes Care 2004; 27: 1047-1053.

2. Organización Mundial de la Salud: WHO, Country and Regional Data. [Acceso 25 de enero de 2008]. Disponible en

http://www.who.int/diabetes/facts/world_figures/en/print.h tml.

3. Snowling N; Hopkins W. Effects of Different Modes of Exercise Training on Glucose Control and Risk Factors for Complications in Type 2 Diabetic Patients. Diabetes Care.2006; 29:2518-27.

4. Boulé N, Haddad E, Kenny G, Wells G, Sigal R. Effects of exercise on glycemic control and body mass in type 2 diabetes mellitus: a meta-analysis of controlled clinical trials. JAMA.2001; 286:1218-1227.

5. Sigal R, Kenny G, Wasserman D, Castaneda-Sceppa C. Phisical Activity/ Exercise and Type 2 Diabetes. Diabetes Care. 2004; 27: 2518-39.

6. Albright A., Franz M, Hornsby G, Kiska A, Marrero D, Ullrich I, et al. American College of Sports Medicine position Stand. Exercise and Type 2 diabetes. Med\&Sci in Sports Exercise. 2000; 32:1345-60.

7. Colberg S.The impact of exercise on insulin action in type2 Diabetes Mellitus: Relationship to prevention and control. Insulin.2006; 1:85-98.

8. Eves N, Plotnikoff R. Resistance Training and Type 2 Diabetes. Diabetes Care. 2006; 29: 1993-1941.

9. Praet S., Van Loon L. Optimizing the therapeutic benefits of exercise in type 2 diabetes.J Appl Physiol.2007; 103: 1113-20.

10. Peirce N. Diabetes y Ejercicio. Br J Sports Med. 1999; $33 ; 161-172$.

11. García de los Ríos M. Guía Clínica Diabetes mellitus tipo 2. Serie de Guías Clínicas Minsal NºXX, $1^{\mathrm{a}}$ edición. Minsal, Santiago, 2006.

12. Golberg L, Elliot D, \& Kuehl K. Assessmnet ok exercise intensity formulas by use of ventilator threshold. Chest. 1988; 94 (1): 95-98.

13. Brzycki M. Strength testing - predicting a one-rep from resp to fatigue. Journal of Physical Educaction, Recreation and Dance. 1993; 64: 88-90. 
PEÑAILILLO, L.; ARMIJO, F.; OSORIO, M.; ROSALES, J. Efectos del entrenamiento aeróbico versus fortalecimiento de sobrecarga muscular en pacientes con Diabetes Mellitus Tipo 2 en un contexto de atención primaria chilena. Rev. Motr. Hum., 11(1): 11-19, 2010.

14. Bompa, Tudor. Periodización del entrenamiento deportivo. Cuarta edición. Barcelona, España. Editorial Paidotribo; 2000.

15. Ross E, Kerr D, Fraccionamiento de la Masa Corporal: Un Nuevo Método para Utilizar en Nutrición, Clínica y Medicina Deportiva. Revista de Actualización en Ciencias del Deporte. 1993; 1(3).

16. ALAD. Revista de la Asociación Latinoamericana De Diabetes. Guías ALAD de Diagnóstico, Control y Tratamiento de la Diabetes Mellitus Tipo 2. 2007.

17. Nguyen N, Rahme E, Dasgupta K. Acute ExerciseInduced Glucose Change During an Exercise Program in Type 2 Diabetes. Jcrpjournal. 2008;28:122-127.

18. Stratton I, Adler A, Neil H, Matthews D, Manley S, Cull C, Hadden D, Turner R, Holman R. Association of glycaemia with macrovascular and microvascular complications of type 2 diabetes (UKPDS 35): prospective observational study. BMJ 2000;321:405-412.

19. McGill M, Felton A. New global recommendations: a multidisciplinary approach to improving outcomes in diabetes. Primary Care Diabetes 2007; 1 (1): 49 - 55.

20. Dunstan D, Daly R, Owen N, Jolley D, De Courten M, Shaw $\mathrm{J}$, et al. High intensity resistance training improves glycemic control in older patients with type 2 diabetes. Diabetes Care. 2002; 25: 1729-1736.

21. Renard E. Monitoring glycemic control: the importance of self-monitoring of blood glucose. AmJMed. 2005; 118(9A):12S-19S.

22. Coutinho M, Gerstein H, Wang Y, Yusuf S. The Relationship Between Glucose and Incident Cardiovascular Events. Diabetes Care.1999; 22:233-240.

23. Giannopoulou L, Ploutz-Snyder L, Carhart R, Weinstock $\mathrm{S}$, et al. Exercise is Required for Visceral fat Loss in Postmenopausal Women with Type 2 Diabetes. J Clin Endocrinol Metab. 2005; 90 (3): 1511-1518.

24. Chan D., Watts G., Barrett P, Burke V. Waist circumference, waist-to-hip ratio and body mass index as predictors of adipose tissue compartments is men. Q J Med. 2003; 96: 441-447.

25. Castaneda-Sceppa C, Layne J, Munoz- Orians L, Gordon $\mathrm{P}$, Walsmith J, Foldvari M, et al. A randomized controlled trial of resistance exercise training to improve glycemic control in older adults with type 2 diabetes. Diabetes Care.2002; 25: 2335-2341.
26. Achten J, Gleeson M, Jeukendrup A. Determination of the exercise intensity that elicits maximal fat oxidation. Med Sci Sports Exerc. 2002;34(1):92-7).

27. Houmard J, Tanner C, Slentz C, Duscha B, McCartney $\mathrm{J}$, Kraus W. Effect of the volume and intensity of exercise training on insulin sensitivity. J Appl Physiol 2004;96:101106.

28. Braun B, Sharoff C, Chipkin S, Beaudoin F. Effects of insulin resistance on substrate utilization during exercise in overweight women. J Appl Physio. 2004.; 197: 991-997.

29. Hawley J, Lessard S. Exercise training-induced improvements in insulin action. Acta Physiol (Oxf). 2008 Jan;192(1):127-35.

30. Röck1 K, Witczak C, Goodyear L. Diabetes, Mitochondria and Exercise. Rev Esp Cardiol. 2008; 8:25-32.

31. Miyazaki Y, Glass L, Triplitt, Wajcberg E, Mandarino L, DeFronzo R. Abdominal Fat Distribution and Peripheral and Hepatic Insulin Resistance in Type 2 Diabetes Mellitus. Am J Physiol Endocrinol Metab. 2002; 283: E1135-E1148.

32. Lastra G, Manrique C, Lastra G. Cardiometabolic syndrome Inflammation, adipose tissue, insulin resistance and atherogenesis the puzzle grows. Acta Med Colomb. 2005; 30 (3):13-19.

33. Wahrenberg H, Hertel K, Leijonhulvud B, Persson L, et al. Use of Waist Circumferenci to Predict Insulin Resístanse: Retrospective Study. BMJ. 2005; 330: 13631364.

34. Rankinen T, Kim S, Pérusse L, Deprés J, et al. The prediction of Abdominal Visceral Fat Level from Body Composition and Anthropometry: ROC analysis. International Journal of Obesity. 1999; 23:801-809.

35. Balkau B, Deanfield J, Despres J, Bassand J, et al. International Day for the Evaluation of Abdominal Obesity (IDEA): a study of waist circumference, cardiovascular disease, and diabetes mellitus in 168,000 primary care patients in 63 countries. Circulation. 2007; 116:1942-1951.

36. Gautier J, Mourier A, Kerviler E, Tarentola A, Bigard A, Villette J, Guezennec C, Cathelineau G. Evaluation of Abdominal Fat Distribution in Noninsulin-Dependent Diabetes Mellitus: Relationship to Insulin Resistance. J Clinical Endocrinology \& Metabolism.1998; 83:1306-1311. 
PEÑAILILlO, L.; ARMIJO, F.; OSORIO, M.; ROSALES, J. Efectos del entrenamiento aeróbico versus fortalecimiento de sobrecarga muscular en pacientes con Diabetes Mellitus Tipo 2 en un contexto de atención primaria chilena. Rev. Motr. Hum., 11(1): 11-19, 2010.

37. Karter A, Mayer-Davis E, Selby J, D’Agostino R, et al. Insulin sensitivity and abdominal obesity in AfricanAmerican, Hispanic, and non-Hispanic white men and women : The insulin resistance and atherosclerosis study. Diabetes. 1996; 45(11): 1547-1555.

38. Azuma K, Heilbronn L, Albu J, Smith S, et al. Adipose Tissue Distribution in Relation to Insulin Resistance in Type 2 Diabetes. Am J Physiol Endocrinol Metab. 2007; 293: E435-E442.

39. Vásquez G, Duval S, Jacobs D, Silventoinen K., Comparison of Body Mass Index, Waist Circumference, and Waist/Hip Ratio in Predicting Incident Diabetes: A MetaAnalysis. Epidemiol Rev. 2007; 29: 115-128.
40. Snijder M, Van Dam R, Visser M, Seidell J. GAT Aspects of body Fat are Particularly Hazardous and how do we measure them. International journal of Epidemiology. 2006; $35: 85-92$.

41. Sigal R, Kenny G, Boulé N, Wells G, Prud'home D, Fortier M, et al. Effects of Aerobic Training, Resistance Training, or both on Gycemic Control in Type 2 Diabetes. Ann Intern Med 2007; 147:357-369.

\begin{abstract}
Compare two training protocols, aerobic and overload, of eight weeks duration in relation to changes in physiological variables and kineantropométricas of adult patients with Type 2 Diabetes Mellitus (DM2) belonging to the Health Center Laura Vicuña, municipality of El Bosque, Santiago.

The sample consisted of 15 subjects randomized into three groups: Aerobic $(n=5)$, which conducted training on stationary bike at a moderate intensity (50-70\% maximum heart rate (HRmax)); overload $(n=5)$, which conducted training to 70-80\% of $1 \mathrm{RM}$; and Control $(n=5)$, which did not make training. Capillary glycemia was tested once a week, before and after each session. variables were evaluated glycosylated hemoglobin ( HbAlc), fasting blood glucose and kineantropométricas at the beginning and end of the intervention. Both training groups were not significant decreases in HbAlc. The aerobic group decreased significantly ( $p=$ 0.04) capillary glucose in your workout, fasting glucose, waist circumference, and a decreased percentage and absolute adipose tissue; the overload group decreased significantly $(p=0,04)$ the adipose tissue in absolute and central skinfolds. All groups increased their muscle mass, to be significant $(p=0.04)$ for the aerobic and control groups.

Only the aerobic group obtained significant changes in both variables related to glycemic control, as body composition and distribution, but when comparing both training groups we can not conclude that a protocol to produce major changes to the other.
\end{abstract}

Key words: Exercise - insulin resistance - glycosylated hemoglobin - glycemic control - waist circumference kineanthropometry.

Dirigir correspondencia a:

Luis Peñailillo Escarate

Avenida El Bosque 1290, Santa Inés, Viña del Mar

CHILE

Fono: (56) 322274397

Fax: (56) 322274395

E-mail: 1.penailillo@ecu.edu.au

RECIBIDO 8-07-2010

ACEPTADO 27-07-2010 